\title{
Research on the Current Situation of Development of Phenomenology and the Influences on Language Cognition
}

\author{
Zhongliang Cui \\ Department of Philosophy Dalian University of \\ Technology, Dalian 116024, China \\ Foreign Language School Chuzhou University , \\ Chuzhou 239000, China;
}

\begin{abstract}
In this paper, we conduct research on current situation of development of phenomenology and the influences on language cognition. Language is a part of human cognition, it not only by the group, including cultural types and communication mode, objective social constraints, as also shared with human cognitive subject and the innate cognitive ability is closely related. Thus, the perspective of the cognitive linguistics and the cognitive introduced for expanding and deepening the research on cognitive linguistics has an important value. From the cognitive perspective and reveals language internalization mechanism is an important path to deepen the study of second language acquisition. So, with multidimensional research value and cognition, can provide important theoretical reference for language internalization. At the same time, the body cognitive is not the purpose of people, but merely a device methods or strategies. That is to say, those who have an information, experience and knowledge module, just as the main body of language cognition of raw materials, they need by the consciousness of main body of the higher order language system to filter, restructuring, deformation and consolidation, and language simulation of this new product for the secondary and experience.
\end{abstract}

Keywords- Phenomenology, Language Cognition, Basic Influences, Current Situation.

\section{Introduction}

Language is the main tool to spread the information, but it is not the only tool. In the people's daily communication, and in the process of spreading information through a variety of

\author{
Huili Wang \\ Foreign Language School, Dalian University of \\ Technology, Dalian 116024, China
}

media, in addition to the use of language, often accompanied by means such as audio and video, only in this way, people can try to find the right information. From the broad sense to explain the "language of the cognitive analysis" that is mainly from the perspective of psychological perception for the analysis of linguistic phenomenon. Put more bluntly, that means the speaker speaking is what kind of the psychological activities made him say that and don't say that, the hearer is what kind of psychological activity makes him listen understand it and don't understand. From this angle analysis explain language phenomena are the "language of the cognitive analysis". We're talking about is the need to pay attention to the cognitive linguistic analysis of a pair of important concepts namely "basic category" and "typical".

Systemic functional linguistics, from the perspective of the social cognitive language system, it is concerned with social communication needs what features and language systems can provide for the user to choose to achieve the communication function of the network system structure component or meaning potential. Its core research is each component of the language system which provides the meaning potential, can accomplish what features of the social communication, users according to the function of the social communication needs to choose in the meaning potential, to achieve social communicative 
functions. Function is the meaning as function system is the semantic system meet the demand for social communication function is formed [1-3].

Phenomenological pedagogy in the significance of education research is more of a performance on its methodological with significance. Why the phenomenological method is applicable to education research and possible, because the phenomenology of concern is the issue of the life world, and the education problem is an important part of the life world. For the education research, therefore, the phenomenological pedagogy is a kind of method, because it does not provide for the final design of the world, also didn't tell people what is the ultimate way of life, of course did not tell people the ideal way of life in where. Before the phenomenology, like other science, psychology, accept the guidance of positivism, and pushed it into the dominant status of methodology. Positivism did promote the birth and development of psychology, however, positivism from the beginning is not accepted by all the psychologists. Conation psychology representative explicitly opposed influenced by positivism content of Wundt's psychology. The later development of psychology also shows that the positivism cannot be as dominant methodology of psychology, as generally guided by positivism structuralism, structuralism and behaviorism has two made psychological crisis. In the face of such facts, Husserl on positivism criticism will naturally cause many psychologists, to shake leading position of positivism in psychology. The following figure one demonstrates the principles.

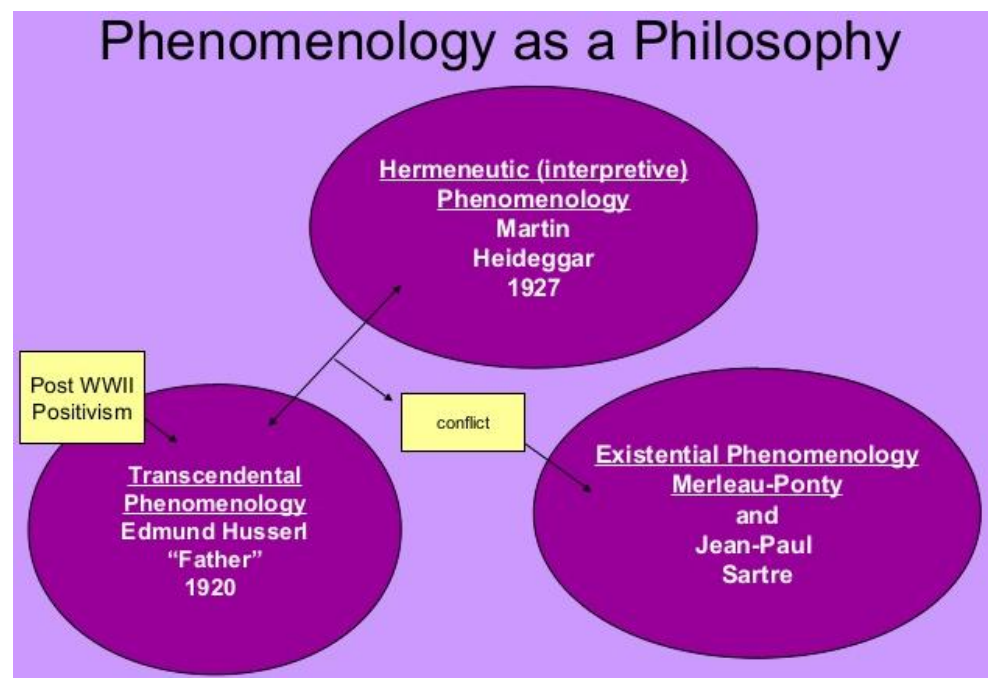

Figure 1. The Primary Components and Architecture of the Phenomenology Theory

In this paper, we conduct research on the current situation of development of phenomenology and the influences on language cognition. Language has a close contact with the human brain activity as process of language learning is a process of human brain activity and the process of mental activity. As linguistics, social linguistics, psychology, cognitive science, and the information processing, and other related disciplines language learning is becoming more and more attention. People begin to realize that in learning a foreign language, the importance of language learning strategies and the cognitive strategies in language learning. In the later parts, we will discuss in detail.

\section{The Proposed Methodology and Perspective}

The Concepts of Phenomenology. Phenomenology of education is the phenomenology of spirit, idea and method to study the phenomenon of education or a school 
of thought education problem. Since at the beginning of its fresh, it with a new vision, clever and wisdom as well as the unique idea of open and leads the people education idea, change and transformation of thinking and research methods of transformation and reconstruction. Especially for education theory research in China, are increasingly forming a stream of irreversible trend and trend of phenomenology of education research and schools of thought contend as is to a certain extent, to promote and deepen the education further spread of the phenomenology of spirit and movement with the radio and transfer [4-5].

The basic task of phenomenology is to grasp the essence of consciousness of law. Husserl thinks only in transcendental reduction were thoroughly the phenomenon, is not mixed any existing factors can be intuitive, are analyzed and described, subjective consciousness can be revealed, to the nature of consciousness. The so-called phenomenological reduction is transcendental the reduction, "called the phenomenology, because it put the world into a phenomenon as is called reduction, because it leads us back to experience the meaning of the world and existence source, back to our experience itself", namely the reduction to the pure subjectivity. Consciousness is the consciousness of people, only the understanding of the subject since the true knowledge paradigm.

Self-awareness as the movement of the double recognition is both for approval and recognition, for approval, only as a recognized it is sure and true self-consciousness, and basic consciousness of the self-consciousness contains the other, it is for his awareness of their own consciousness, and thus potentially self-consciousness is the consciousness of the entity, it on his own, consciousness and the awareness of its physical nature, only to the entity from the body, from simple main body to the body of the entity that also need through double recognition activities of self-awareness to deduce.
Ecological phenomenology criticism the subject-object dualism and the separation of fact and the value, and carefully examine the possibility of it instead of the modern philosophy. With ecological phenomenological movement, the position of two rival discernible: Nietzsche and Husserl's followers put forward the natural ethics realism, they think no matter good or bad things are ultimately the truth itself, value should be based on the ethical facts closer. Ecology phenomenology editor believes, for our own understanding to be close to the change of the environment, as well as the various practical decision making, will be started in solid and discerning clear, this is clear for our own ethics and the metaphysical presupposition and surrounding environment, it will not only on individuals but also on the culture about our current behavior. Let's start with ecological phenomenology "metaphysical" principle. According to this principle, crossing the phenomenological methodology of traditional can acquire a basic consistency: all phenomenological position seeks to negate the mind the separation of the world called the principle of "metaphysics" is potentially misleading, because from the beginning of Husserl, phenomenon scientists have described without through the lens of metaphysics foreseen before experience. Under this condition, the object is to phenomenon, namely to present things in the consciousness. Researchers don't assume that the object does not exist as don't assume that this related theory is not true just tries to mount on them. In the process of suspension, asking people to give up on things before see, prejudice and attitude to things. The whole world by the parentheses, placed in behavior, requires people to learn to face the thing itself directly, in a new, pure, broad perspective, that from a purely or transcendental ego of examining phenomenon again [6].

The Language Cognition. Language is an autonomous system and users cannot depend on the language and the objective world and exist 
independently, so the purpose of language cognition is to found the system's structure composition and the components how to constitute the language symbol system. The meaning of the language is not a structural linguistics focuses on the objects. About the meaning of the language as the theory is that the meaning of a word in the language of the internal relationship between the network structures as the value of a word is its differences with other words in the system which could be reflected from the figure two.

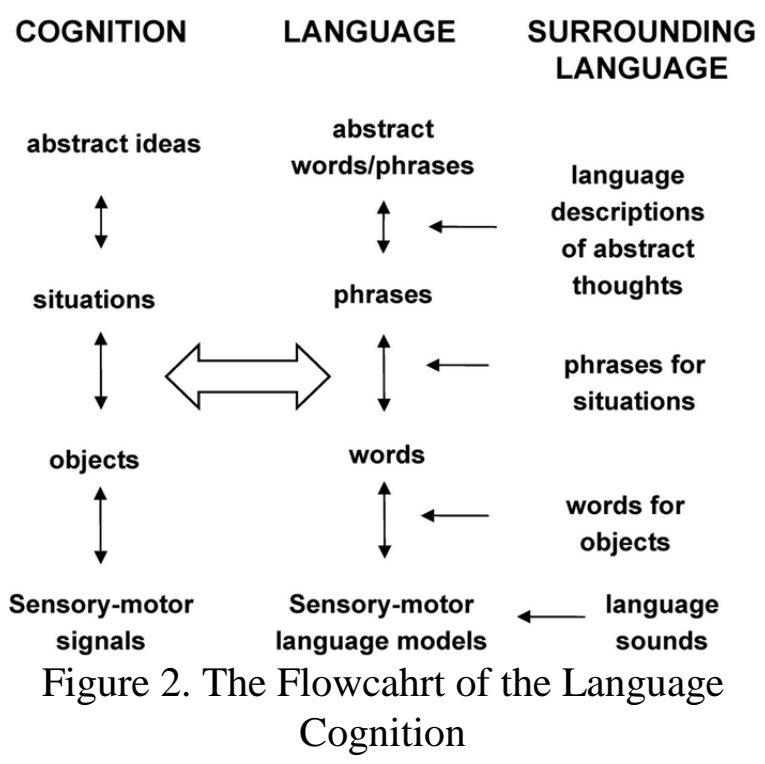

Background of the structuralism is scientism. Scientific socialism pursuit of the pure objectivity, resolutely eliminate the influence of the subjective factors and the role of people. According to this principle, only is not affected by the user and the real world of the study of language system is the objectivity, that as a result, the structuralism language system as the research object, and not pay attention to the use of the language users and language environment. Structural linguistics is the pure language study, it isolates the language and all other relevant factors, the only language system as the research object, regardless of language and relationship between the user and social culture, also don't consider how to produce meaning. It studies is the language of closed system and the characteristics of the system itself and cognitive research present two different trends: one is the theoretical research of trend micro and concrete. Separated from philosophy epistemology and cognition, the former belongs to the category of cognitive theory, was later introduced other different disciplines and more in-depth theoretical study, the thesis into specific and in-depth; Second, and cognition to the experimental and the applied research and cognition as a set of unique cognitive model combined with the practice of different areas that play high application value, and provides guidance for the application of different cognitive domain which could be summarized as the following aspects [7].

- In Heidegger here, although the ontology language to performance through people's language, but not all people speak language can reveal existence directly, the reason is that language has both reveal the value of existence but also has the function of cover is Heidegger don't think need to communicate and follow the logic of the public and syntax of the language can reveal the existence, only from the ontology and liberated from the grammar to a more primitive structure of language to reveal the nature of the existence.

- Whether the growth of the psychological reality or of the declarative nature of paradigm of an orderly, logical, physical reality is people use language symbols such as system construction in his mind, language is the foundation of human subjectivity and the sole condition of human subjectivity is possible and applicable.

- Development of deconstruction, Lacan about floating can refer to the theory, and the sliding, on the grounds of eternal participation of people don't think in the language origin of absolute truth. It will be the meaning of the language to the infinite, so that people in the clear logos 
centralism absurd vanity comprehend the richness of language and meaning of unlimited.

The Linguistic Theories. Structural linguistics with particular emphasis on primary systematic, distinguish between language and speech, believed that language is essentially a kind of the symbol system, the combined with the pronunciation and meanings of a structure, the nature of language elements depends on the relationship between system elements; Language of systemic performance for combination relations and paradigmatic relations both crisscrossed relationship; Language has the synchronic and diachronic, pay attention to the synchronic study of language and linguistics should be descriptive rather than normative [8].

Language sense is the earliest research method is to use sound recorder to record and analyze the output of the language information, the latest research techniques should belong to the photography camera technology as people use the technology to record the dynamic changes of the pronunciation. In terms of language awareness, context is the most important influence its conditions. In the research of language signal, mostly associated with aims of acoustic characteristics, vowels and consonants. Impact on the language sense is the most important areas of study of the speech perception model, namely, motor model, synthetic analysis model, fuzzy logical model, model and rules. Chomsky founded the transformation of the generative linguistics is the philosophical basis of the Cartesian rationalism thought, think that all language knowledge from the rational, the language is essentially refers to the ability to abstract the language user rather than the language of the specific observable behavior, it is not possible by observation and analysis of the external facts of language behavior. He thinks that the human brain are born with an innate language acquisition capacity, only need to study as a linguist exists in the human brain is used to generate the internal language fact those rules.

\section{Conclusion}

In this paper, we conduct research on the current situation of development of phenomenology and the influences on language cognition. Cognitive linguistics, and other branches of linguistics, has its own unique discipline target and character, describe and explain the language system, structure and the function, and how these features are language systems. From this angle to study language, therefore, conceptual model, language provides a window to study cognitive function, understand the nature of language, structure, and the organization of the thought. Cognitive linguistics is different from other language research train of thought of is the language used to reflect the basic nature and the essential characteristics of the human mind. Our research proposes the novel paradigm for the corresponding research which holds special meaningful and significance.

\section{Acknowledgement}

This paper is financially supported by the key project of the humanities and social science research in colleges and universities in Anhui: Research on the Wittgenstein approach of the cognitive linguistics and cognitive science of complementary (NO. SK2015A432).

\section{References}

[1] Husserl, Edmund. Cartesian meditations: An introduction to phenomenology. Springer Science \& Business Media, 2012.

[2] Alloul, Adam, et al. "FeynRules 2.0-A complete toolbox for tree-level phenomenology." Computer Physics Communications 185.8 (2014): 2250-2300.

[3] Kadastik, Mario, et al. "Implications of the $125 \mathrm{GeV}$ Higgs boson for scalar dark matter and for the CMSSM phenomenology." Journal of High Energy Physics 2012.5 (2012): 1-17. 
[4] Raison, C. L., and A. H. Miller. "Role of inflammation in depression: implications for phenomenology, pathophysiology and treatment." (2013): 33-48.

[5] Alarcón, Jose Manuel, J. Martin Camalich, and J. A. Oller. "Improved description of the $\pi \mathrm{N}$-scattering phenomenology at low energies in covariant baryon chiral perturbation theory." Annals of Physics 336 (2013): 413-461.

[6] Nelson, B., et al. "What are the neurocognitive correlates of basic self-disturbance in schizophrenia?: Integrating phenomenology and neurocognition: Part 2 (Aberrant salience)." Schizophrenia research 152.1 (2014): 20-27.

[7] Grant, Jon E., Liana RN Schreiber, and Brian L. Odlaug. "In Review Phenomenology and Treatment of Behavioural Addictions." Canadian Journal of Psychiatry 58.5 (2013).

[8] Divotgey, Florian, Lisa Olbrich, and Francesco Giacosa. "Phenomenology of axial-vector and pseudovector mesons: decays and mixing in the kaonic sector." The European Physical Journal A 49.10 (2013): 1-9. 